\title{
Lutte contre les inondations de la Siagne Programme de mise en sécurité des personnes et des biens
}

\author{
Siagne floods prevention \\ A program to make people and goods safe
}

\author{
par G. Cina, \\ Président du Syndicat Intercommunal de la Siagne et de ses Affluents (S.I.S.A) \\ J.M Sotty, I.P.G.R. \\ F. Calvet, SAFEGE-CETIIS
}

The Districts of the Siagne watershed, coastal river flowing into the Mediterranean sea, along the border of the Var and Alpes Maritimes departments, suffered from numerous important floods. Aware of their responsibilities and due to great development stakes on their area, they have chosen to build a complete security system for people and property settled in areas liable to be flooded. Once actually set up in the District Association of the Siagne River and Tributaries (SIS.A), the Owner, it will include :

- A warning system, based on the mastered use of the available images, processed by Meteo France.

- An operational structure able to be used depending on different levels (watch, vigilance, pre-alert, alert) and different levels of seriousness, whose missions will be clearly defined. It will be based on GIS, to keep an operating knowledge data base.

- A coordination, on one hand, with the Departmental Security Planning and, on the other hand, with the Districts Security Planning.

The S.ISA. now deals with the set problems by econonomic and operating integration into a recent Districts Association.

\section{LE CONTEXTE}

Le bassin versant de la Siagne s'étend sur environ $512 \mathrm{~km}^{2}$, en limite des départements du Var et des Alpes Maritimes.

Elle se jette dans la Méditerranée entre Cannes et Mandelieu, où elle traverse une ancienne zone de delta, à faible pente et fortement anthropisée.

Les communes de son bassin versant ont connu à plusieurs reprises des problèmes d'inondation dus aux débordements de la Siagne et de ses affluents. Afin de répondre de façon adaptée à ces problèmes, elles se sont progressivement regroupées sous la forme d'un "Syndicat Intercommunal de Défense Contre les Inondations de la Siagne" (S.I.S.D.I.S).

Initialement composé des communes de la partie aval, le S.I.S.D.I.S., qui a réalisé de nombreux aménagements de protection contre les inondations (recalibrage de tronçons, redimensionnement d'ouvrages, etc.), a reçu l'adhésion des communes situées dans les parties moyennes et amont du bassin versant et a pris le nom de Syndicat Intercommunal de la Siagne et de ses Affluents.

A la suite d'une démarche de réalisation d'un atlas départemental des zones inondables, dont les résultats ont été communiqués aux communes concernées, les services de l'Etat ont programmé des actions (PPRi) visant à imposer aux communes des prescriptions en matière d'occupation du sol dans les zones inondables, en particulier dans la basse vallée.

Au-delà de l'intégration au POS, ces démarches avaient également pour objectif d'inciter les communes à prendre en compte le risque d'inondation dans leurs politiques de développement.

Ces actions, tout en remettant fortement en cause les projets de développement envisagés par les communes concernées, s'attachaient à prévoir les conséquences des projets dans le futur, mais ne proposaient cependant pas de solutions pour les personnes et les biens déjà installés dans les parties basses de la vallée, mais laissaient cependant la porte ouverte 


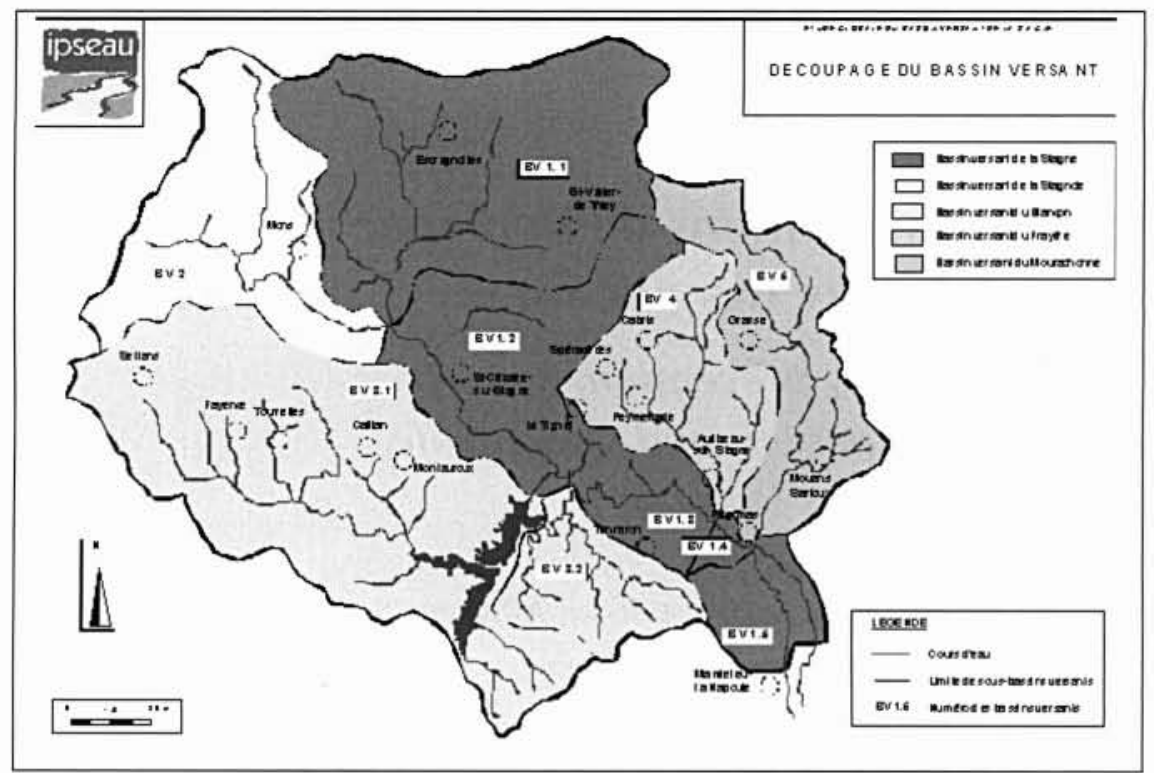

1. Bassin Versant de la Siagne (Origine IPSEAU) .

à la mise en place d'un système d'alerte aux crues dans une optique de mise en sécurité des personnes exposées.

La mission de conception d'un tel système a été confiée à I'I.P.G.R. (Institut de Prévention et de Gestion des Risques urbains) à Marseille, en partenariat avec NICAYA (société de conseil qui a pour vocation de faciliter l'intégration des dimensions humaines et culturelles dans les pratiques techniciennes).

\section{II — LES DIFFÉRENTES ÉTAPES DE LA CONCEPTION}

L'I.P.G.R. a proposé au S.I.S.A. une mission en deux étapes :

- La réalisation d'un Audit de la situation actuelle destiné à préparer la phase de conception en analysant :

- Les différents points de vue (Etat, collectivités, riverains) sur les risques d'inondation dans la vallée, sur les mesures à prendre pour se protéger, afin d'évaluer en quoi et comment le système de prévision et d'alerte peut y contribuer.

- La faisabilité du système d'alerte et son intégration au sein des différentes communes du SISA, par une analyse des aspects techniques, opérationnels, organisationnels et réglementaires.

- La conception du système proprement dit.

\subsection{L'audit}

Il a conduit à un diagnostic de la situation actuelle, ainsi qu'à une analyse des points forts et des points faibles définissant des pistes de progrès et des marges de progression, portant sur :

\section{- La connaissance du risque,}

Le bassin versant de la Siagne a fait l'objet de plusieurs études hydrologiques et hydrauliques, permettant de préciser la nature des aléas, les types de réponses envisageables en matière d'aménagements, et les préconisations possibles en matière de droit du sol, mais ces études sont essentiellement centrées sur la partie aval du bassin versant. De plus, ne concernant que la gestion du droit du sol, elles ne traitent que la crue centennale et n'abordent pas la question de la vulnérabilité. Elles ne sont pas adaptées à la gestion de crise.

Il sera donc apparu nécessaire de disposer d'études plus adaptées à la problématique de la gestion de crise, d'une cartographie d'aléa sur l'ensemble des sous-bassins versants de la Siagne, pour différentes classes d'événements pluvieux. Ces études d'aléa devraient permettre d'évaluer les dommages potentiels sur l'ensemble de la zone, afin de définir des stratégies d'intervention et des plans de secours cohérents et efficaces.

- Les réseaux de mesure et la prévision hydro-météorologique,

Plusieurs opérateurs (Météo-France, la DIREN, EDF dans le cadre de l'exploitation du barrage de St Cassien) effectuent différents types de mesures et prévisions hydrologiques sur le bassin versant. La commune d'Auribeau gère un réseau de mesure (pluviomètres et limnimètre), limité à la Frayère, dans une optique de mise en sécurité des personnes.

Un radar du réseau Aramis est en cours d'installation dans le Var, à proximité du lac de St Cassien, conçu pour couvrir la majorité des bassins du département des Alpes Maritimes.

La société Dimensions a implanté un réseau d'antennes SAFIR, qui peut potentiellement être utilisé, en appoint de l'imagerie Radar, sur la quasi-totalité de la région PACA.

Ces réseaux ne sont pas conçus pour effectuer une prévision hydro-météorologique et ne couvrent pas l'ensemble du bassin versant. Les prévisions de Météo-France sont réalisées à l'échelle régionale et ne sont donc pas suffisamment précises pour être utilisées dans le cadre d'un dispositif de mise en sécurité des personnes.

Il sera donc nécessaire de "contracter" avec Météo-France dans le cadre d'une convention spécifique, d'utiliser les nouvelles possibilités offertes par l'installation du Radar, ainsi que d'intégrer, dans les outils proposés par Météo-France, l'imagerie SAFIR.

\section{- L'alerte hydro-météorologique,}

L'alerte est de type classique (B.R.A.M.) et les procédures de diffusion classiques, via le C.I.R.C.O.S.C., le Centre Météorologique Interrégional du Sud-Est à Aix (C.M.IR.S.E) 
suit plus spécifiquement certains événements sévères, mais ces informations restent à la disposition des professionnels qui en font la demande.

Une organisation existe donc, mais il n'y a pas de structure d'annonce de crues sur le bassin-versant. L'Etat n'envisage pas d'en installer une. Les B.R.A.M. ne sont pas suffisamment territorialisés. Les collectivités n'ont pas organisé de système d'astreinte hydro-météorologique $24 \mathrm{~h} / 24$. sauf pour le sous-bassin versant de la Frayère. Elles ne savent pas vraiment "quoi faire" des informations plus ou moins précises qui leur sont communiquées. Le problème de "digestion" et de "traduction" de l'information reste entier.

Elles doivent en effet être pertinentes avec l'échelle du bassin versant (quelques centaines de $\mathrm{km}^{2}$ ) et les échéances souhaitées (quelques heures).

Il sera donc utile de fiabiliser les informations qui servent à déclencher l'alerte, de les "pré-traiter" afin de les rendre exploitables par les collectivités et leurs partenaires, enfin d'organiser la veille, la pré-alerte, l'alerte et la gestion de crise de façon cohérente avec les missions des différents partenaires (Météo-France, collectivités, services locaux, départementaux et interrégionaux de la Sécurité Civile et le cas échéant le public.

Il sera indispensable que l'ensemble du dispositif d'alerte soit adapté aux temps de réponse des sous-bassins (45 minutes à 1 heure).

\section{- L'organisation des secours,}

A l'exception de deux communes (Mandelieu et Cannes) les communes du bassin ne sont pas dotées d'un Plan Urbain de Secours d'Urgence (P.U.S.U.) et s'en remettent à l'organisation des sapeurs-pompiers en cas de crise.

Outre leurs procédures d'intervention classiques, les services de la Sécurité Civile disposent d'un Plan départemental spécial inondations.

Les P.U.S.U. ne sont pas spécifiques aux inondations. Ce sont avant tout des plans généraux de moyens et pas des plans d'intervention.

Les plans ont été conçus pour être curatifs et n'ont donc pas vocation à anticiper sur les événements.

Il sera, en conséquence, judicieux de prévoir d'étendre la démarche "P.U.S.U." à d'autres communes du bassin en la complétant par une définition des plans d'intervention.

Compte tenu des temps de réponse des sous-bassins, cette approche devra être complétée par une organisation intercommunale de veille, pré-alerte et alerte capable de mobiliser, en fonction de seuils prédéterminés, l'ensemble des acteurs, selon des procédures préétablies.

\section{- Les structures de pilotage et d'interface,}

Jusqu'à présent, la problématique de mise en sécurité des personnes face au risque d'inondation n'a pas fait l'objet d'une approche globale regroupant l'ensemble des partenaires concernés.

Il sera donc nécessaire de construire un plan d'intervention en identifiant chaque acteur nécessaire et en précisant ses missions pour chaque maillon du processus de mise en sécurité des personnes, en matière de :

- Prévision et mesures hydro-météorologiques pour la météorologie et l'hydrologie,

- Alerte hydro-météorologique pour la veille, l'interprétation de la situation, le déclenchement de l'alerte, la diffusion de l'alerte et le suivi de la situation.
- Pilotage des opérations de mise en sécurité préventives des personnes pour la prise de décision par le maire et la mise en œuvre de ses décisions par les services communaux et les services de la Sécurité Civile,

- Plan de secours pour le pilotage des interventions, la coordination, la réalisation des interventions par les trois acteurs concernés, les collectivités, la Sécurité Civile, le public.

L'audit a également permis, malgré la mise en évidence de trois entités culturelles ayant des rapports fortement différenciés à l'eau et à leurs rivières dans le bassin versant (la basse vallée, partie prenante de la Cote d'Azur, la moyenne vallée, centrée sur l'industrie des parfums autour de Grasse, la haute vallée dédiée au tourisme vert) de dégager un consensus sur les conditions de faisabilité, articulé de la façon suivante : - Pour être crédible et fiable, ce dispositif doit être mené dans le cadre d'une approche globale,

- Il s'agit de la première étape d'un processus beaucoup plus vaste de prévention et de gestion des risques d'inondation. Sa conception et sa réalisation doivent prendre en compte les impératifs de développement économique auxquels les communes sont confrontées aujourd'hui,

- Le système doit être réaliste, opérationnel, approprié aux besoins et attentes des différents partenaires.

\subsection{La démarche proposée}

Elle intègre l'ensemble des pistes de progrès explorées dans le cadre de l'audit et comporte :

- La mise en place d'un système de prévision hydrométéorologique,

- L'installation d'un réseau de télémesure (pluie et niveaux),

- La réalisation d'un plan d'intervention destiné à mettre en cohérence alerte, plans de secours communaux et départementaux.

- La construction d'un Système d'Information Géographique.

\subsection{Le système de prévision hydro-météorologique}

Le système choisi comportera :

- Un bulletin spécialisé édité chaque jour et disponible sur minitel, comportant sur une zone précise (par exemple la haute vallée de la Siagne, ou la basse vallée), une prévision à $24 \mathrm{~h}$ de la hauteur de pluie, de son heure probable de début et de fin, assorti d'une information sur la qualité de la prévision.

- Une assistance téléphonique en temps réel par le prévisionniste de Météo-France,

- Une station de travail HP équipée du système ASPIC de Météo-France, qui intègre, sur un même écran HP relié par une ligne spécialisée au C.M.I.S.E et à Cannes, l'ensemble de l'imagerie disponible (infrarouge, satellite, mosaïque radar, lames d'eau, Safir), ainsi que les données de pluie délivrées par les stations automatiques voisines.

Ce système ne sera complètement opérationnel qu'après la mise en service du radar varois, prévue pour 2001.

Il sera complété par une convention spécifique de dialogue avec le prévisionniste local de Météo-France.

\subsubsection{Le réseau de télémesure.}

Il s'agit d'une simple extension du réseau mis en place par la commune d'Auribeau, constitué de : 
- Un poste central équipé du logiciel "LOGEMAC 2000" de la société INOVAREX (ex-AUTEG),

- Trois stations "NIVEMAC" gérant des capteurs de niveau,

- Une station "PLUIEMAC" pour un pluviographe. Son rôle sera surtout important après le déclenchement de l'alerte car il permettra de suivre au sol l'évolution des phénomènes, tout en constituant progressivement une base de connaissance du fonctionnement de la rivière et de ses affluents.

\subsubsection{Le Plan d'Intervention}

Il devra permettre de répondre aux questions suivantes :

\section{- Où intervenir ?}

En évaluant les débits de débordement dans chacun des sous-bassins versants et en proposant l'un d'entre eux comme indicateur de débordement. Le débit de débordement, le débit correspondant à l'exutoire du sous-bassin et la lame d'eau correspondant à ce débit seront également définis.

En cartographiant l'aléa décennal, et l'aléa exceptionnel, à l'aide de modèles rustiques. Cette cartographie "orientée Plan d'intervention" est sensiblement différente, en termes de précision des cartographies d'aléa destinées au P.O.S. Elle doit en effet permettre de repérer les axes préférentiels d'écoulement, d'identifier les personnes résidant dans la zone à risques, ainsi que les équipements vulnérables et/ou stratégiques.

\section{- Quand intervenir ?}

La méthode reposera sur l'organisation d'une veille permanente, qui alimente en informations la structure décisionnelle. Celle-ci enclenche un processus d'action à trois degrés correspondant à une montée en puissance de l'organisation (banal, grave, catastrophique) :

- Mise en vigilance à $\mathrm{H}$ (heure prévisible du début d'un événement) $-24 \mathrm{~h}$; la procédure de suivi précis de l'événement est activée. Cette mise en vigilance est déclenchée par le bulletin minitel personnalisé diffusé par Météo-France. - Franchissement d'un seuil à $\mathrm{H}-1$ h. La cellule de crise est en place et se prépare à agir selon les procédures prévues (locales ou en concertation avec le Préfet), en fonction du niveau de gravité prévu,

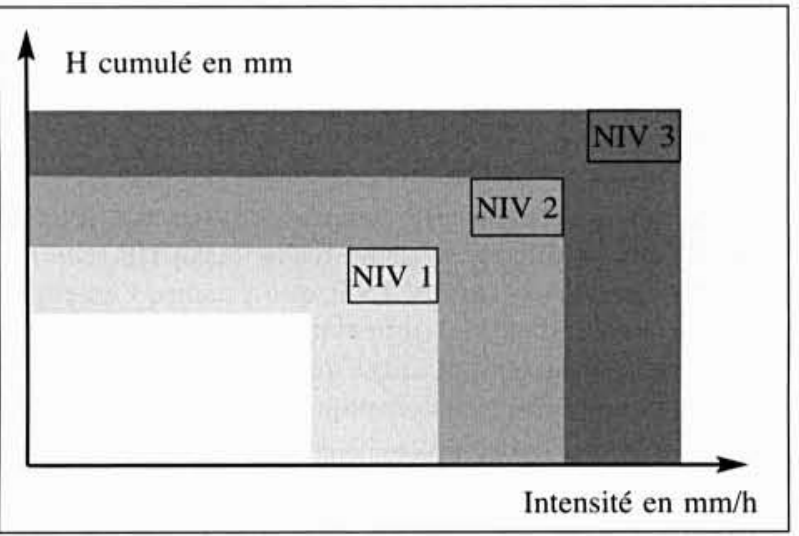

- Franchissement d'un seuil d'alerte lorsque l'événement est là. L'action est déclenchée.

L'outil de prise de décision aura alors la forme suivante :

- Mise en vigilance. Niveau 1

- Mise en pré-alerte. Niveau 2

- Mise en alerte. Niveau 3

Lorsque le seuil de vigilance est franchi, le suivi précis de l'événement est enclenché.

Lorsque le seuil de pré-alerte est franchi, la structure décisionnelle est mobilisée.

Lorsque le seuil d'alerte est franchi, la structure décisionnelle informe et lance le processus d'intervention sur le terrain.

En attendant la mise en place du radar, la détection de ces seuils pourra se faire par approche qualitative à partir d'interprétation d'imagerie et de dialogue avec le prévisionniste Météo-France local.

Lorsque les informations radar seront disponibles, la prévision pourra être plus précise et sera faite sur la base d'un outil de prévision, dessiné ci-dessus.

Cet outil, qui permet de positionner un épisode pluvieux, réduit à ses deux principaux paramètres, sera établi pour chaque sous-bassin, par rapport aux seuils précédemment définis.

\section{- Avec quels moyens ?}

Seront construits :

- Des plans de moyens communaux, c'est-à-dire des listes exhaustives des moyens matériels (hébergement, restaurants, hôpitaux, entreprises travaillant de façon courante dans la commune avec leurs moyens spécifiques, etc.), humains (médecins, secouristes, etc.) et financiers.

- Des plans d'intervention, c'est-à-dire une stratégie d'intervention, interfacée avec le PSS départemental, une déclinaison en procédure-type y compris pour les fonctionnements dégradés, chacune d'entre elles étant définie, acteur par acteur, sous forme de fiche-réflexe.

- Un système d'appel téléphonique en masse, hiérarchisant ses appels en fonction des trois niveaux (vigilance, alerte, pré-alerte) et des trois degrés de gravité (débordement. décennal, exceptionnel).

\subsubsection{Le Système d'Information Géographique}

Il permettra de disposer d'un fond de plan homogène, aisément mis à jour, sur tout le territoire du S.I.S.A.

Cet outil pourra également être utilisé en temps réel pendant la crise, en vidéo-projection.

\section{III - CONCLUSION}

Le S.I.S.A. a donc choisi de se doter d'outils sophistiqués, à la mesure de ses enjeux de développement. Il est maintenant confronté à l'intégration de ce nouvel outil, avec les problèmes particuliers que cela pose (compétences nouvelles, formations, astreintes toujours délicates à concevoir et faire vivre, partage clair des responsabilités), dans une structure intercommunale de création récente. 